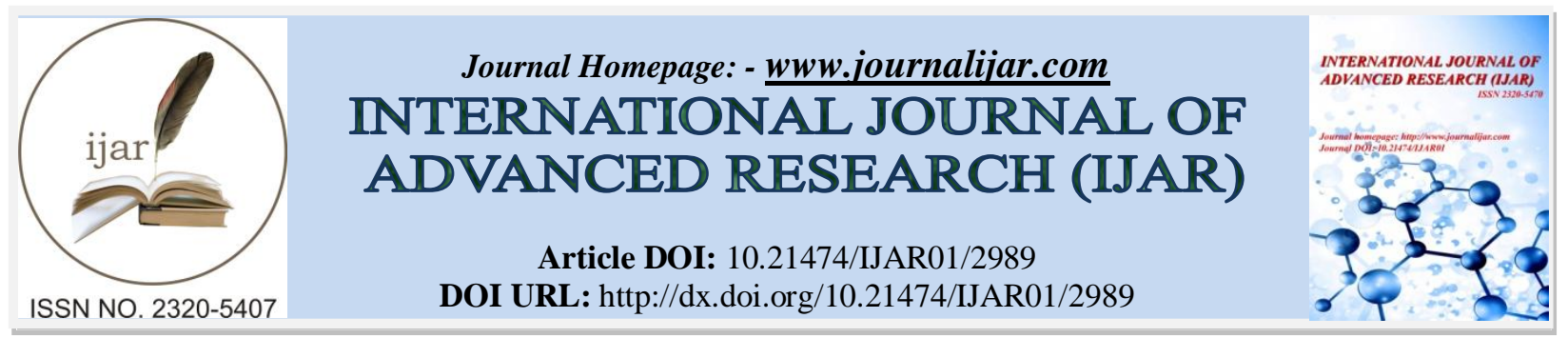

RESEARCH ARTICLE

\title{
PROTECTIVE POTENTIAL OF CYNODON DACTYLON EXTRACT ON LAMOTRIGINE INDUCED BRAIN TOXICITY IN MICE FETUSES.
}

Sharda Singh and S.K. Pandey.

Department of Anatomy, Institute of Medical Sciences, Banaras Hindu University, India

\section{Manuscript Info}

Manuscript History

Received: 28 November 2016

Final Accepted: 27 December 2016

Published: January 2017

Key words:-

antioxidant, Cynodon dactylon, fresh

juice, lamotrigine, teratogenic

\section{Abstract}

Lamotrigine (LTG), an antiepileptic drug believed to be safe in pregnancy whereas Cynodon dactylon (CD) is a herb having antioxidant potential, used for the treatment of various diseases including epilepsy in alternative system of medicine. The study was planned to observe the effect of LTG alone and with Cynodon dactylon on growing brain of mice. Lamotrigine was given orally at a dose of $60 \mathrm{mg} / \mathrm{kg}$ body weight on $7^{\text {th }}-9^{\text {th }}$ gestational day, to the pregnant mice of treated group I (T I) whereas both Lamotrigine $\left(60 \mathrm{mg} / \mathrm{kg}, 7^{\text {th }}-9^{\text {th }}\right.$ day) and Cynodon dactylon juice $\left(0.3 \mathrm{ml}, 6^{\text {th }}-17^{\text {th }}\right.$ day of gestation) were given to treated group II (T II). Control group (C) received the same volume of distilled water during $6^{\text {th }}-17^{\text {th }}$ day of gestation. On $18^{\text {th }}$ day of gestation fetuses from all groups were collected and kept in $10 \%$ formalin for fixation. After three days of fixation the brains were removed from the cranial cavity and observed macro and microscopically. Brain of T I group showed insignificant decrease in weight. The haemorrhagic patches and the prominent blood vessels on various surfaces of brain have been observed in T I group. The macroscopic findings in T II group brain were similar to the control group. The microscopic features of T I brain revealed destruction and oedematous appearance of parenchyma of hippocampus, loss of normal architecture of cortical and subcortical zone, dilated ventricles with disruption of ependymal lining, degeneration and clumping of choroid plexus as compared to control group. Treated group II showed reduction in pathological findings which were appeared in treated group I. Therefore there may be possibility that Cynodon dactylon (CD) fresh juice neutralised the effect of lamotrigine neurotoxicity in growing brain upto certain extent. This may happened due to antioxidant property of CD.

Copy Right, IJAR, 2016,. All rights reserved.

\section{Introduction:-}

Lamotrigine (LTG) [3,5-diamino-6(2,3dichlorophenyl)1,2,4triazine], is an anticonvulsant drug used in the treatment of epilepsy and bipolar disorder in the United States as an adjunctive treatment since 1994 and as monotherapy since 1998 (Goel P et al, 2012). Lamotrigine is used frequently in pregnancy as many workers claimed its malformation rate same as in normal population (Vajda et al, 2006; Richens A., 1994) but others found LTG to produce intra uterine growth retardation and other congenital malformations (Padmanabhan et al, 2003; Morrow et 
al, 2006) and a few studies reported that LTG possess teratogenic action on fetal brain (Marchi et al, 2001; Manent et al, 2008; Mohanty et al, 2011). Cynodon dactylon (CD) is a herb having antioxidant property, used in treatment of various diseases including epilepsy in ayurvedic system of medicine. The present experimental study was conducted to observe the effect of Cynodon dactylon fresh juice on the teratogenic changes in foetal brain, induced by lamotrigine.

\section{Materials and Methods:-}

Expermental animal: Pregnant Swiss albino mice were used as experimental animal for the present study, each weighing 20-25 g. Mice were caged in air conditioned animal house of Department of Anatomy, Institute of Medical sciences, Banaras Hindu University, Varanasi, India.

\section{Methods:-}

Female mice were transferred to cage containing adult males of proven fertility overnight for mating and were inspected next morning for the presence of vaginal plug. The day of vaginal plug positive was considered as gestational day (GD) 0. Pregnant mice were divided into two groups (6 mice in each group), i.e. Control and treated. The treated group further subdivided into two i.e. treated group I (T I) and treated group II (T II). T I group was given Lamotrigine, orally through gavage tube at a dose of $60 \mathrm{mg} / \mathrm{kg}$ body weight from GD 7 to 9 . T II group were treated with $0.3 \mathrm{ml}$ of Cynodon dactylon extract (fresh juice) from day 6 to 17, as well as lamotrigine from 7 to 9 day of gestation. Cynodon dactylon extract is freshly prepared everyday by dissolving calculated amount of lyophilized juice in distilled water. Control group was given distilled water for same duration through same route. On the $18^{\text {th }}$ day of gestation the mice were sacrificed by cervical dislocation. The foetuses were collected by laparotomy and fixed in $10 \%$ formalin for 2 to 3 days. After fixation, the brain were removed from the cranial cavity and examined for gross abnormalities, if any, then weight was measured and finally the organ was processed for microscopic study. The study protocol was approved by animal ethical committee, BHU. All procedures were in accordance with the guidelines of the Animal Welfare Act. Statistical analysis was done by using one way ANOVA to compare the mean weight of control and treated brains of different groups.

\section{Results:-}

As compared to control group, the brain of both T I and T II showed reduction in weight, that was statistically insignificant. The mean weight of the control group was $0.0614 \mathrm{~g}$ (SD .0127), whereas in T I group $0.0599 \mathrm{~g}$ (SD = $0.0062) \&$ in T II group .0604 ( $\mathrm{SD}=.0045)$. On macroscopic examination the outer surface of the growing brain of $\mathrm{T}$ I group revealed prominent dilated blood vessels and haemorrhagic patches, whereas T II group showed the normal appearance of the brain as shown in control group (Fig 1). There was no significant difference in the length and breadth of any group of brain. On microscopic examination hippocampus and cortical zones showed the normal layering pattern in control group whereas T I group revealed destruction of hippocampal region and cortical zone along with oedematous appearance. T II group showed the less destruction of the normal pattern of the layering and disappearance of the oedematous spaces (Fig. 2, Fig.3). In higher magnification the cells of the cortical zone of T I showed the proliferation of the immature growing cells whereas in T II there was no any indication of proliferation of cortical cells but degenerative changes has been observed (Fig. 4). The ventricle of T I group revealed dilatation, destructed choroid plexus and thickening of proliferated immature growing cells in the periventricular area whereas the T II group showed the appearance of ventricle and periventricular area as in control group (Fig.5). In higher magnification the T I group showed the destructed ependymal lining of the ventricle along with the disintegration of choroid plexus whereas T II group showed the of lining ependymal layer of the ventricle at few places as compared to control group.

\section{Discussion:-}

The present study was mainly focussed on the effect of Cynodon dactylon on teratogenic action induced by LTG in growing brain. The mean weight of brain was found decreased in T I group as compared to control, although it was not statistically significant. Haemorrhagic patches on the surfaces of brain and prominent dilated blood vessels, especially on basal surface were also seen in the present study. Mohanty et al, (2011) observed that the brain weight of growing foetuses exposed to LTG was found increased but, not significant statistically. They also reported that some of the LTG exposed foetus had well defined dark brown swellings over parieto-occipital region of head (exencephaly) and hemorrhages of various sizes over the body (C. Mohanty et al, 2011). The mice brain was used for the present study whereas rat brain was used for the previous study. Marchi et al, (2001) mentioned that fetuses of the LTG treated group had reduced body weight at birth, increased volume and diameter of the cerebral structure 
(Marchi et al, 2001). Another study also reported no significant difference in mean brain weight and mean brain volume in control and experimental rat foetuses (Sah N et al, 2013). Further it was observed in the same report that one of the lamotrigine exposed rat fetus had exencephaly whereas no any such case was found in the present study. Microscopic study in present work showed derangement of architecture and interruptions in the continuity of the cortical layers, oedematous appearance in subcortical and periventricular zone, dilatation of ventricles and disruption of ependymal lining along with destruction and clumping of choroid plexus have been observed in the brain of treated group I fetuses. A similar finding was reported by Marchi et al (2001), who revealed that the use of lamotrigine during the organogenesis period was associated with histological alterations. They analyzed cortex, subcortex, ependyma and lateral ventricles in their study on rat brain, after giving LTG on 9-11 day of pregnancy. Their results revealed the subcortical density enhancement, and ventricle dilatation (Marchi et al, 2001). In another study it was observed that the LTG treated growing brain showed dilated ventricle with less differentiated cortical layers (C. Mohanty et al, 2011). Treatment of pregnant wistar rats with a high dose of LTG ( $20 \mathrm{mg} / \mathrm{kg} /$ day) resulted in clear morphological alterations in the parietal cortex and in CA1 and CA3 hippocampal field (Manent et al, 2008). Histological study of the cerebral cortex revealed ill defined plexiform layer and dilated lateral ventricle (Sah $\mathrm{N}$ et al, 2013). Excess oxidative stress may be one of the mechanisms that contribute to teratogenicity. The nervous system is particularly vulnerable to the deleterious effects of reactive oxygen species (Puchchakayala G, 2012). Cynodon dactylon has been proved for its neuroprotective activity against aluminium induced toxicity (Sumathi T et al, 2011). The methanolic extract of Cynodon dactylon, given orally was found to significantly increase the levels of superoxide dismutase, catalase and NADH dehydrogenase in comparison with the control group (Manju Bhaskar et al, 2015). C. dactylon has been proved to inhibit lipid peroxidation in CNS (Auddy B et al, 2003; Rai PK, 2010). Correlating our findings with the previous works we can interpret that free radical scavenging activity of Cynodon dactylon may be the responsible for the prevention of oxidative stress induced teratogenecity. Goodman et al, 1996 showed that pre-treatment with either estrogen or progestins could prevent excitotoxic and oxidative stress injury and enhance cell survival in cultures of embryonic hippocampal neurons. Roof, R.L. et al. 1992 have been showed that the high endogenous levels of progesterone in female rats dramatically reduced cerebral oedema. So the alternative hypothesis responsible for the neuroprotective effect of CD in LTG induced abnormalities growing brain might be presence of steroids in it.

\section{Conclusion:-}

Lamotrigine treatment followed by supplementation of Cynodon dactylon resulted in reduction in macro and microscopic neuropathological findings. It is therefore possible that the antioxidant activity of Cynodon dactylon neutralised the effect of lamotrigine induced changes in various parts of growing brain and helps to prevent the teratological changes in brain cell and tissue.

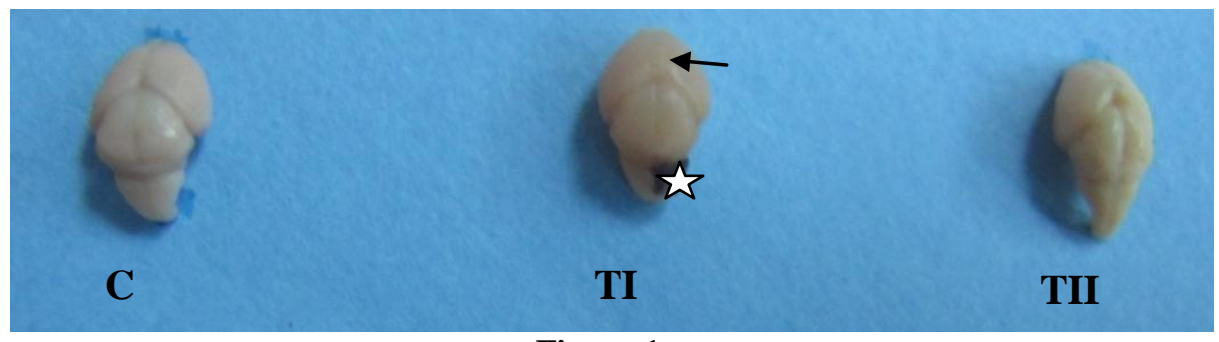

Figure 1:-
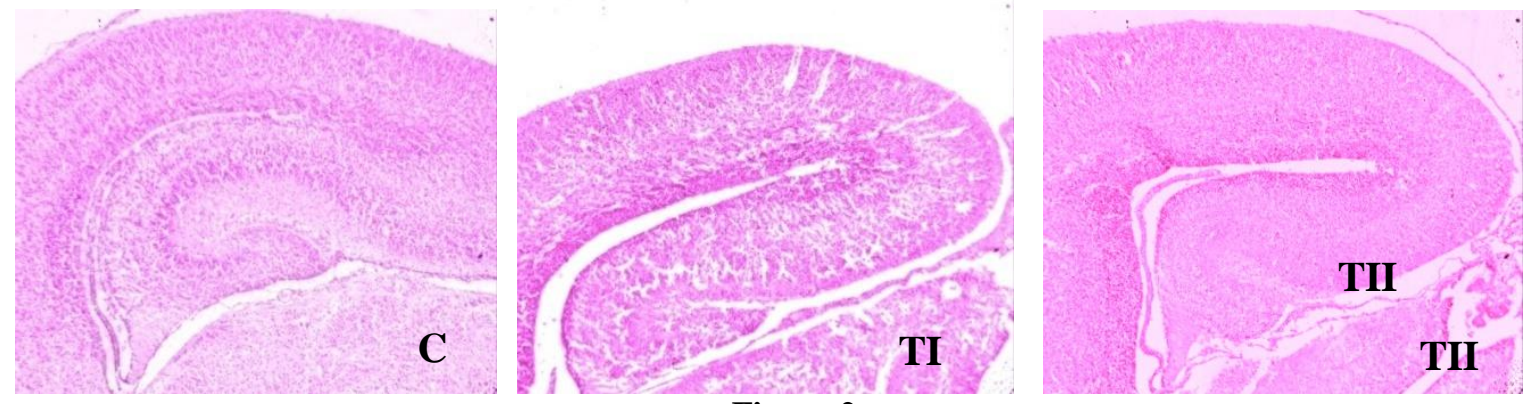

Figure 2:- 

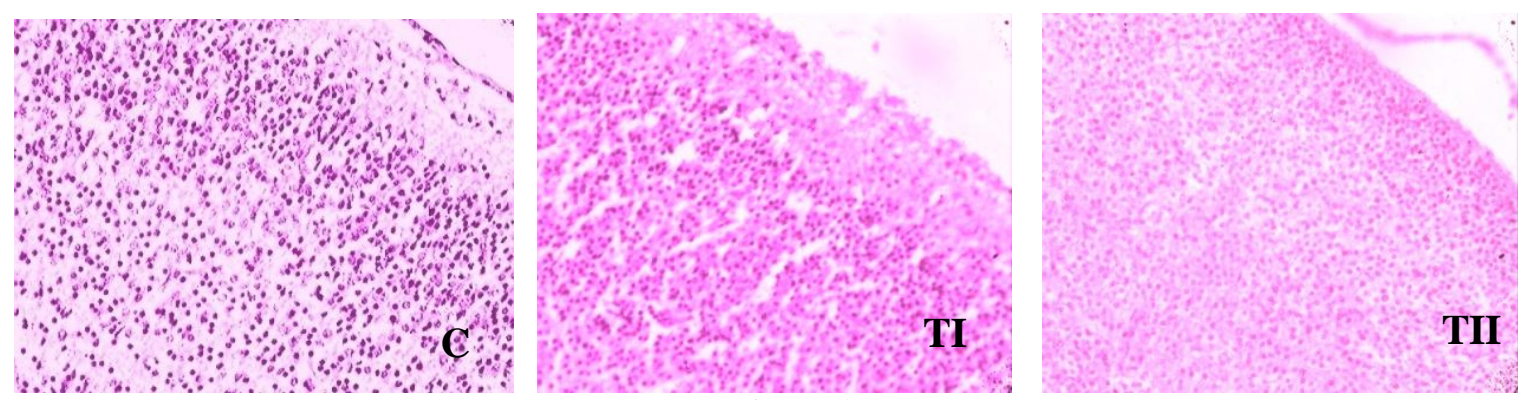

Figure 3:-
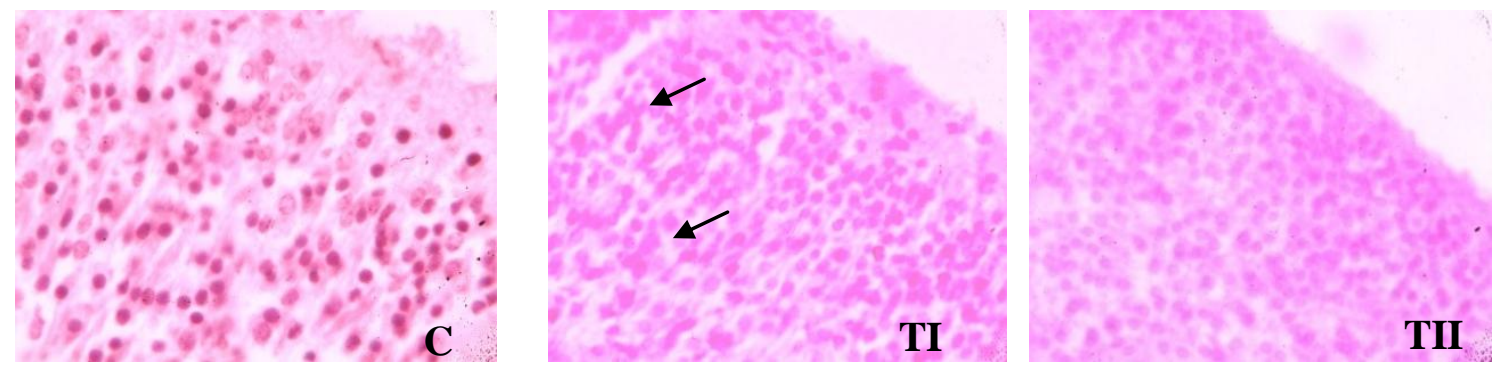

Figure 4:-
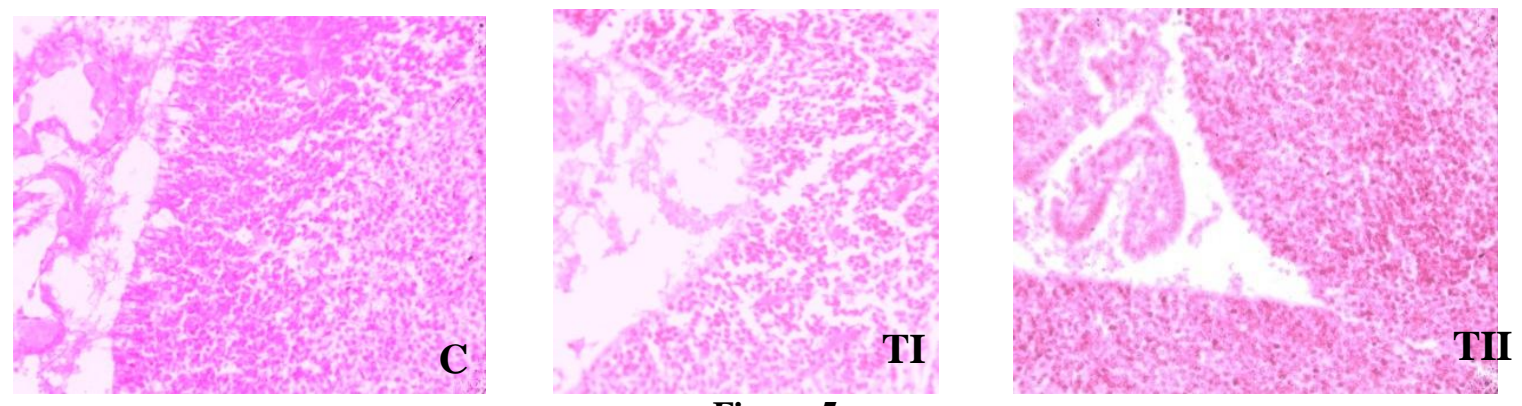

Figure 5:-

\section{Legends of Figure:-}

Figure 1: Photograph showing dorsal surface of fetal brain of control (C) and treated groups (TI \& TII). T I group showing haemorrhagic patch $(\widetilde{\zeta})$ and prominent vessels $(\sim)$, whereas T II group showing normal appearance of dorsal surface of the brain as seen in control group.

Figure 2: In low magnification (H\&E, 100X), photomicrograph of brain showing features of hippocampus. T I group showing destruction and oedematous appearance of parenchyma of hippocampus, whereas T II showing the normal features of hippocampus as seen in control group.

Figure 3: In high magnification (H\&E, 400X), photomicrograph of brain showing layers of cortex. The control brain (C) reveals normal pattern of the various layers of cortex T I group showing destruction of the various cortical layers and oedematous spaces, whereas the T II group showing only the mild destruction of cortical layers as compared to control group.

Figure 4: In oil immersion magnification (H\&E, 1000X), photomicrograph of brain showing cortical layers of control (C) and treated groups (TI \& TII). T I group showing the degenerated growing cells of various layers of the cortical zone along with the oedematous appearance and clumping of degenerated cells at places ( $\sim$ ), whereas T II group showing the normal appearance of the cells as seen in control group.

Figure 5: In high magnification (H\&E, 400X), photomicrograph of brain showing ependymal lining and choroid plexus, of control (C) and treated groups (TI \& TII). T I group showing the destruction of ependymal lining and 
clumping of degenerated choroid plexus and T II group reveals the normal appearance of region as seen in control group.

\section{References:-}

1. Bhaskar, M, Chinmaykinkar and Grewal H. (2015): Neuroprotective effect of Cynodon dactylon and Phyllanthus amarus on the antioxidant defense system in Alzheimer's disease. Indo American Journal of Pharmaceutical Research, 5(1): 338-343

2. Goel,P., Isher,H, Saha,P.K., Tendon R., Devi L., Dhami,G.P. (2012): Lamotrigine Induced Hypersensitivity Syndrome in Pregnancy. Nep J of Obs and Gynae, 7 (1), 13: 68-70

3. Goodman, Y.,Bruce A.J., Chenq, B.,Mattson M.P. (1996) Estrogens attenuate and corticosterone exacerbates excitotoxicity,oxidative injury, and amyloid beta-peptide toxicity in hippocampal neurons. J. Neurochem, 66: $1836-1844$

4. Manent,J.B., Jorquera,I., Franco,V, Ben-Ari Y., Perucca, E., Represa, A.(2008): Antiepileptic drugs and brain maturation: Fetal exposure to lamotrigine generates cortical malformations in rats. Epilepsy Research, 78(23):131-139

5. Marchi,N.S.A. de, Azoubel, R., Tognola W. A.( 2001): Teratogenic Effects of Lamotrigine on Rat Fetal Brain A morphometric study. Arq Neuropsiquiatr, 59 (2B): 362-364.

6. Mohanty, C., Shah, N., Dhungel, S., Das B.K. (2011): Effect of Lamotrigine on Fetal Rat Brain. People's Journal of Scientific Research, 4(2): 5-7

7. Morrow, J., Russell, A., Guthrie, E., Parsons, L., Robertson, I., Waddell, R., Irwin, B., Mc Givern, R.C., Morrison, P.J., Craig, J.( 2006): Malformation risks of antiepileptic drugs in pregnancy: a prospective study from the UK epilepsy and pregnancy register. Journal of Neurology, Neurosurgery and Psychiatry, 77(2): 193198.

8. Padmanabhan, R., Abdulrazzaq, Y.M., Bastaki, S.M., Shafiulla M., Chandranath, S.I. (2003) Experimental studies on reproductive toxicologic effects of lamotrigine in mice. Birth Defects Research Part B, Developmental and Reproductive Toxicology, 68(5): 428-438.

9. Puchchakayala G, Akina S, and Thati M. (2012): Neuroprotective Effects of Meloxicam and Selegiline in Scopolamine-Induced Cognitive Impairment and Oxidative Stress. International Journal of Alzheimer's Disease, 2012: 1-8.

10. Rai, P.K., Jaiswal, D., Rai, D.K., Sharma, B., Watal, G, (2010): Antioxidant potential of oral feeding of Cynodon dactylon extract on diabetes-induced oxidative stress. J Food Biochem, 34: 78-92

11. Richens A. (1994): Safety of lamotrigine. Epilepsia, 35(5): 37-40.

12. Roof, R.L., Stein D.G.(1992): Progesterone treatment attenuates brain edema following contusion injury in male and female rats. Restor. Neurol. Neurosci., 4: 425-427

13. Sah,N., Pandit ,R.K., Dhungel S. (2013) : Effect of Lamotrigine on fetal rat brain morphology. Janaki Medical College Journal of Medical Sciences, 1(1): 26-29

14. Sumathi, T., Shobana C., Kumari, B.R., Nandhini, D.N. (2011): Protective role of Cynodon dactylon in ameliorating the aluminium-induced neurotoxicity in rat brain regions. Biol Trace Elem Res., 144: 843-853

15. Vajda F.J., Hitchcock A., Graham J, Solinas,C., O’Brien,T.J., Lander C. M. , Eadie M.J . (2006): Foetal malformations and seizure control: 52 months data of the Australian Pregnancy Registry. Eur J Neurol, 13: 64554. 